\title{
Prediction of Topside Electromagnetic Compatibility in Concept Phase Ship Design
}

\author{
Ajmal Gharib, Hugh D. Griffiths, Fellow, IEEE, and David J. Andrews
}

\begin{abstract}
An approach for prediction of topside electromagnetic compatibility in the concept phase of naval ship design is proposed and demonstrated. The approach was developed by utilizing the commercially available numerical computational package, Computer Simulation Technology (CST), to assess the electromagnetic environment of a Royal Navy Type 22 Batch II Frigate. A number of the results of such an assessment were validated using measurements on a 1:50 scale copper model of the Type 22 Batch II Frigate. The approach was then applied to a new concept phase design study for a Future Patrol Ship (FPS), produced by UK Ministry of Defence's Naval Design Partnership. This work is expected to be useful in assessing the severity of topside EMI, using numerical modelling and simulations, in Concept Phase Ship Design.
\end{abstract}

Index Terms - EMI, EMC Concept Phase Ship Design, Modelling and Simulation

\section{INTRODUCTION}

A Naval ship is a complex system of systems which may be deployed to accomplish certain strategic and defense missions. In order to achieve its objectives effectively, a naval ship may employ armament and electromagnetic (EM) sensors, such as radars and other systems many of which use antennas. It is essential for a naval vessel to be able to identify who and where its enemies are and then to take appropriate actions, such as attack or evasion. Nowadays, all of these are done by utilizing powerful and sophisticated electronic sensors and systems [1].

Thus the topside EM environment of a naval vessel is dense, containing a plethora of EM sensors used for the purposes of communication, navigation, detection, homing, direction finding and tracking [1], [2]. Each of the sensors is required to offer certain capabilities based on the ship's roles and missions. Since the topside of a naval ship contains a limited space [3], the antennas have to be located in close proximity to one another. Shipboard transmitters are required to emit high power, perhaps several tens of Megawatts of Effective Isotropic Radiated Power (EIRP), in order to detect and track distant targets, while at the same time topside receivers are required to be highly sensitive, within a few $\mathrm{dB}$ of -174 $\mathrm{dBm} / \mathrm{Hz}$ thermal noise, in order to detect weak echoes from distant targets [4]. Topside sensors, such as radars, may radiate signals at harmonics or other spurious frequencies, out of band, in addition to their fundamental frequencies [5]. If the frequency of an undesired signal falls within the intermediate frequency (IF) bandwidth of a victim receiver, electromagnetic interference (EMI) may result - depending on the exact signal levels [2]. EMI may also occur due to intermodulation distortion in which two or more signals mix in the receiver, producing intermodulation products: more than just the sum and difference of the input signals [6]. The occurrence of EMI depends on the power of the signal going through the receive system, as a high powered signal may saturate one or more of the receive system devices, such as mixer or amplifier, if the magnitude of the signal exceeds the compression point for one of those devices [7], [8].

Topside interference may cause performance degradation of the on-board sensors, by preventing the reception of the desired signals through a reduction in signal to noise ratio (SNR), and if this is extended to one or more systems they could then be rendered operationally unavailable. EMI reduces the ability of a naval vessel to be an effective combatant and makes it more prone to incidents and vulnerable to attacks [9]. The consequence of not addressing EMI issues in time can be potentially catastrophic as evidenced by HMS Sheffield in May1982 [10].

Designing a naval vessel with a reduced level of EMI helps in ensuring that downstream in the design process there are fewer interference issues to be tackled. As a result, emphasis has been placed in this study upon discovering and alleviating EMI problems in the concept phase of ship design, the phase when the ship designers produce initial estimates of the ship's dimensions, displacement, stability, survivability and hydrodynamic performance. From an iterative process, the concept ship design team evolve an appropriately balanced design [11] with emphasis on maximising overall ship performance [12]. However, as part of the concept design the main elements of the equipment, such as radar systems, satellite communication systems, electronic warfare systems, communication systems, navigational systems and identification friend or foe systems, are selected for the design. Therefore, there is a need for the electromagnetic environment to be assessed. However, for UK naval combatant design, this is done well after the early fleet and in very early concept ship design studies, while the ship configuration choices are still very fluid. Thus, should electromagnetic compatibility (EMC) problems be discovered (often when the ship gets to sea or very late in acquisition when a physical model is produced), it is necessary for the designers to modify the design until an acceptable level of EMC is achieved. This can be very disruptive late in the design and, since most of the ship design is fixed, the remedial measures are highly constrained [11].

Thus, one of the main challenges encountered by ship designers in the concept phase of ship design is the ability to allocate topside sensors in a way such that the various systems coexist harmoniously and perform their desired operations, 
without causing mutual interference or adversely affecting each other's operations. The very complex and non-linear nature of the EMI problem makes it difficult for ship designers to accurately predict the magnitude of the interference problems between the topside antenna systems, without using suitable tools which demand a high level of definition - not available in concept design. Past EMI data from similar ships or comparable EM sensors on different naval vessels may be useful in terms of being aware of which of the two or more topside sensors may interfere with each other but it is unlikely to be sufficient. This is because any difference between current ships and future concept based design configurations such that the parameters of the shipboard antenna systems in question, differ even for similar ships, could then significantly change the topside EM environment.

This paper shows how this problem can be addressed using a suitable computational electromagnetic (CEM) tool to model the ship and its topside EM environment and then to simulate the EM interactions of the antennas against each other on the concept level ship definition in order to quantify the interference between them. The numerical simulation package CST was employed in which each of the topside antennas is modelled based on its operating parameters. After achieving the required representations for each of the antennas, the antenna models were simulated on the ship model, using the two approaches, outlined in Section III, to determine the coupling between them. If, for example, the interference between two topside antennas is predicted to be above a selected tolerable level, the antennas can be relocated on the ship, consistent with other ship design constraints, and the simulation is then rerun. This process is repeated until a configuration is achieved where the severity of the interference is reduced to a level that is acceptable to ship designers [2].

\section{EMI COUPLING}

EMI coupling is the transfer of EM energy from the terminals of a transmitting antenna into the terminals of a receiving antenna. EMI coupling in the far-field region of the antenna depends on the gain of the transmitting antenna, gain of the receive antenna and free space propagation losses, and it is given by [13], [14]

$$
\begin{gathered}
C(f, t, d, p)=G_{t}(f, t, d, p)+G_{r}(f, t, d, p) \\
-L_{p}(f, t, d, p)
\end{gathered}
$$

where

$C(f, t, d, p)$ is the coupling between a transmitter and receiver, as a function of frequency (f), time (t), distance

(d) and polarization (p)

$G_{t}(f, t, d, p)$ is transmit antenna gain in the direction of the receiver

$G_{r}(f, t, d, p)$ is receive antenna gain in the direction of the transmitter

$L_{p}(f, t, d, p)$ represents propagation losses.

The interference margin (IM) can be used to determine whether an undesired transmitting signal can cause intolerable level of interference in a receiver. This is done by comparing the power available in a receiver's input terminal to the power required to cause interference in that particular receiver.
The available power at the input terminals of a receiver can be written as [13]

$$
P_{A}(f, t, d, p)=P_{t}(f, t)+C(f, t, d, p)
$$

where $P_{A}(f, t, d, p)$ is the power available in the input terminals of a receiver and $P_{t}(f, t)$ is the transmit power.

For interference to occur, the power required to cause EMI, $P_{i}(f, t)$, must be greater than the power available in the input terminal of the receiver. Therefore,

$$
P_{i}(f, t)>P_{A}(f, t, d, p)
$$

IM is obtained by subtracting the power required to cause interference from the power available in the input terminal of a receiver.

$$
I M=P_{A}(f, t, d, p)-P_{i}(f, t)
$$

All these parameters are measured in $\mathrm{dB}$.

If IM is positive then there is potential for an interference problem. Negative IM indicates no or very little potential for interference.

\section{DETERMINING SHIPBOARD ANTENNA COUPLING}

The first step taken in this work in determining topside antenna coupling was to create virtual representations for the ship and its on-board suite of antennas using CST. The next step was to simulate the EM effects of those antennas on the ship, using an appropriate CEM tool, in order to determine the severity of topside antenna interference.

Using CST Microwave Studio (MWS), shipboard antenna coupling is computed using either its Transient (T) or Asymptotic (A) solver. The choice of the solver depends on the highest operating frequency of the antennas on the ship under consideration. This is because simulation of shipboard antennas operating at upper Ultra High Frequency (UHF) or Super High Frequency (SHF) band, such as radar and satellite communication antennas, on a 130 meter long naval ship using the T-solver of CST MWS with its associated hexahedral meshing can be enormously computationally intensive, due to many-billions of mesh cells that are generated, thus requiring hundreds of gigabytes of computer memory and many weeks of continuously running the solver. This problem is therefore addressed by using the A-solver of CST MWS for simulating shipboard antennas operating at UHF or SHF frequency bands and the T-solver of the same tool for simulating the low frequency operating antennas, such as communication antennas, as outlined in the subsequent subsections of this paper.

\section{A. Time Domain Antenna Model Coupling Calculations}

The T-Solver in CST MWS calculates the antenna coupling over broadband frequency range using hexahedral meshing system. This approach can calculate in a single simulation the antenna coupling between numerous low frequency operating antennas on the ship, such as HF whip antennas.

In Figure 1, a CST model for the Future Patrol Ship (FPS) is shown. The model was produced using Perfect Electrical 
Conductor (PEC), representative of the metallic material, such as steel which has a very smalls electrical resistivity $(0.70 \times$ $10^{-6}$ to $1.16 \times 10^{-6} \Omega \mathrm{m}$ ) [15], used on the superstructure of the actual physical ships. The use of PEC allows the worst case topside coupling scenarios to be assessed, due to its ability to thoroughly reflect incident EM waves from the structure [2].

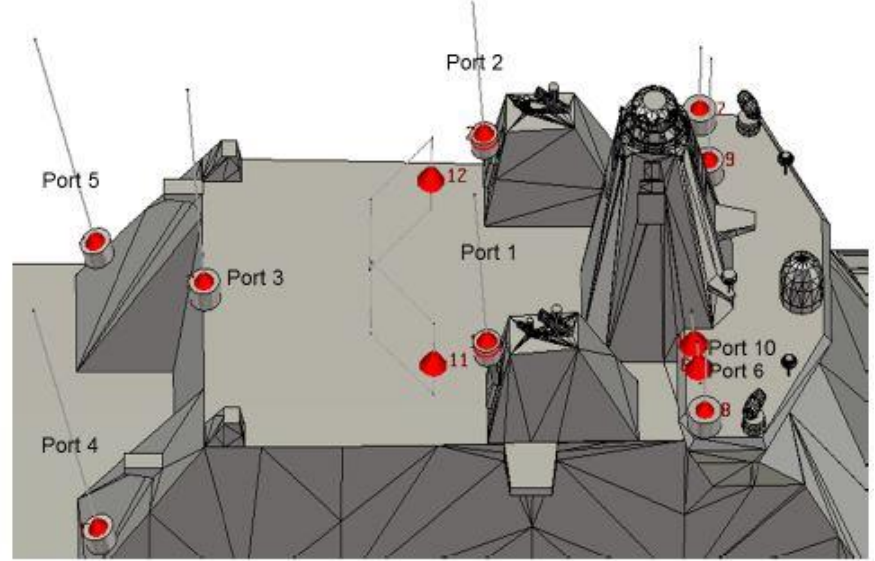

Fig. 1. The various numbered antennas operating at High Frequency (HF) and lower part of Very High Frequency (VHF) on the FPS, shown on the CST model of the main superstructure.

On the FPS CAD model, 10 whip antenna models, marked 1 to 10 , and two skywave loop antenna models, marked 11 and 12, are shown. After the desired reflection coefficient for each of the different antennas on the ship model was determined, the combined ship and antenna models were simulated in CST MWS using the T-solver.

The coupling between each of the different pair of antennas, shown in Figure 1, is calculated over a broadband range of frequency. For example, the coupling between Port 3 and Port 5 antenna models, both transmitting and receiving, is given in Figure 2. When Port 3 antenna model transmits, the level of coupling to Port 5 antenna model is $-26.3 \mathrm{~dB}$. Likewise, when Port 5 antenna model transmits the level of coupling to Port 3 antenna model is $-14.1 \mathrm{~dB}$.

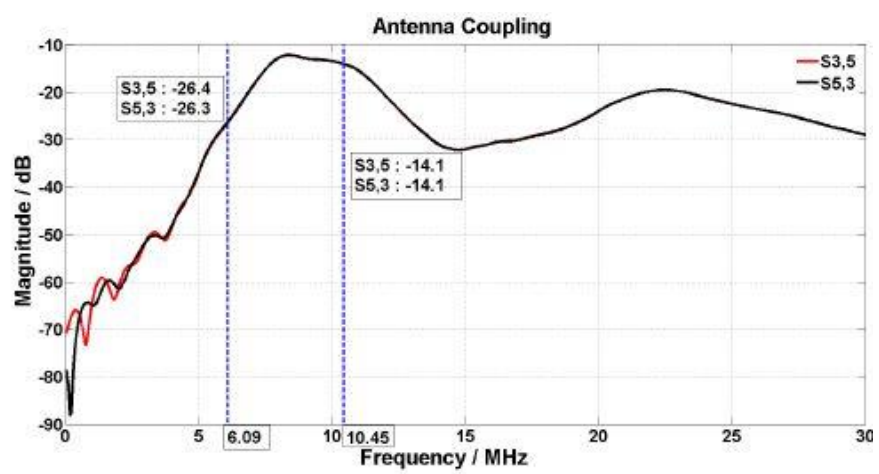

Fig. 2. Antenna coupling between Port 3 and Port 5 antenna models, as analysed by the T-Solver of the CST MWS for the FPS topside model in Figure 1.

The above approach can be utilized in order to compute the magnitude of the antenna interference between the various HF and VHF antennas on a naval vessel. Should the level of interference be severe, the antennas could be relocated on the ship model in order to achieve acceptable level of interference.

\section{B. Time Domain Near-field Coupling Calculations}

For near-field coupling calculations, the near-field effects for each of the antennas can be captured using near-field sources. The field distributions outside the near-field sources are identical to those of the actual antennas [16]. The nearfield sources are imported on to the ship model, produced in CST MWS based on the external dimensions of the physical ship, and allocated in the exact places of the corresponding antennas. The T-Solver or Transmission Line Matrix (TLM) solver of the CST MWS can then be used to simulate the nearfield coupling effects of each of the antennas on to other antennas. The choice of the solver depends on the operating frequencies of the corresponding antennas. The T-Solver can be used in the case of low frequency operating antenna sources while the TLM solver, due to its ability to allocate fine mesh close to the antenna near-field sources and coarser mesh elsewhere, can be utilized for simulating radar near-field sources.

In Figure 3, four near-field sources are shown, marked 1 to 4.

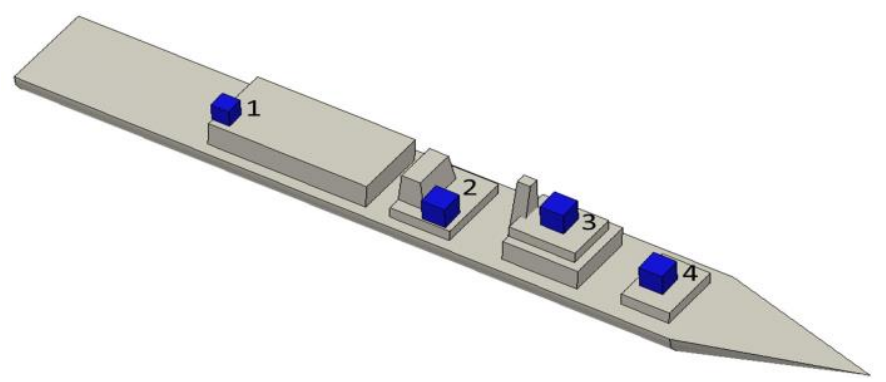

Fig. 3. Near-field sources on a Type 23 ship model.

The near-field coupling effects of antenna 1 was simulated and the result is shown in Figure 4. The level of coupling to other near-field antenna sources is indicated by the amount of surface current that falls on the locations of each of those antennas [16].

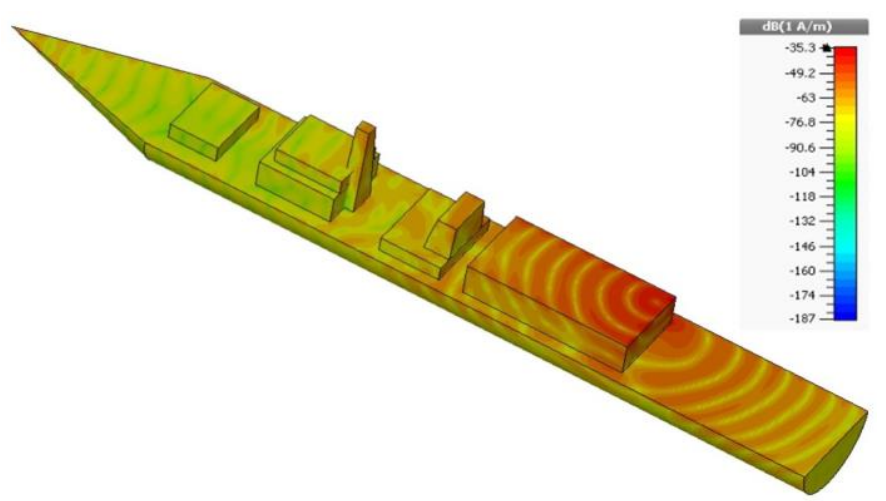

Fig. 4. Distribution of surface current due to emissions of near-field source 1 . 


\section{Frequency Domain Coupling Calculations}

This approach had been used to calculate shipboard antenna coupling between the UHF and SHF operating antennas, such as satellite and radar antennas. The approach has been crossvalidated against the Time Domain coupling calculation approach, see Section V of this paper, and it has been found to yield similar coupling results to that of the Time Domain coupling calculation approach.

For instance, the asymptotic approach has been utilized to calculate the coupling between an INMARSAT $\mathrm{C}$ transmit antenna model, operating at $1645 \mathrm{MHz}$, and an element of the Iridium array satellite receive antenna model, operating at $1623 \mathrm{MHz}$, that is prone to directly receiving EM waves from the mainlobe of the INMARSAT $\mathrm{C}$ transmit antenna and therefore susceptible to interference. The set-up of the radiating sources, prior to running the simulation, is shown in Figure 5. The far-field source on the upper part of the mast represents an element of the Iridium satellite receive antenna array model, while the one on the mast sponson represents the INMARSAT C transmit antenna. Each of the far-field sources was produced for each of the different antennas in CST MWS before they were imported on to the ship model and placed in their designated locations. The far-field sources replace the antenna models as they act as equivalent sources to them [17]. Asymptotic based simulation was then run on the combined ship and equivalent antenna models, using the far-field radiation patterns as excitation sources for the simulation.

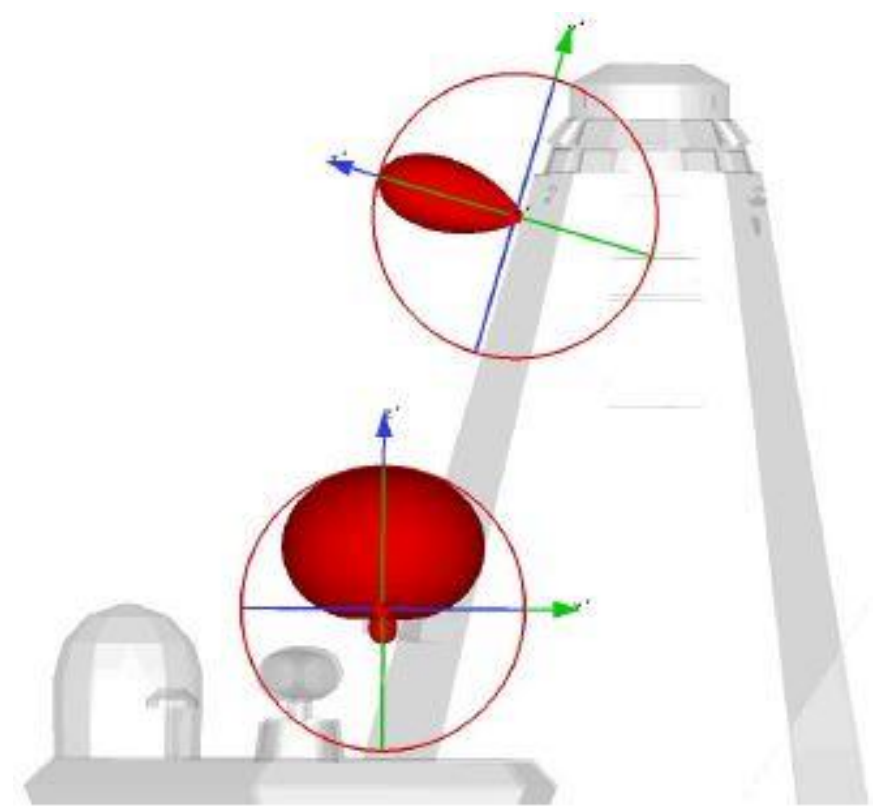

Fig. 5. Far-field antenna sources for INMARSAT C transmit antenna model and an element of the Iridium array satellite receive antenna model on the FPS model.

After completion of the simulation, the resultant far-field sources (RFFSs) which take the effects of the structure into account are automatically calculated by the A-Solver. The RFFSs were then used in CST 2013 Macro to calculate antenna coupling. Using the CST Macro, the coupling between the INMARSAT $\mathrm{C}$ transmit antenna model and an element of the Iridium array satellite receive antenna model was calculated, as shown in Figure 6. The level of coupling to an element of the Iridium array satellite receive antenna model, operating at $1623 \mathrm{MHz}$, when the INMARSAT C transmit antenna model, operating at $1645 \mathrm{MHz}$, transmits is $-62.5 \mathrm{~dB}$.

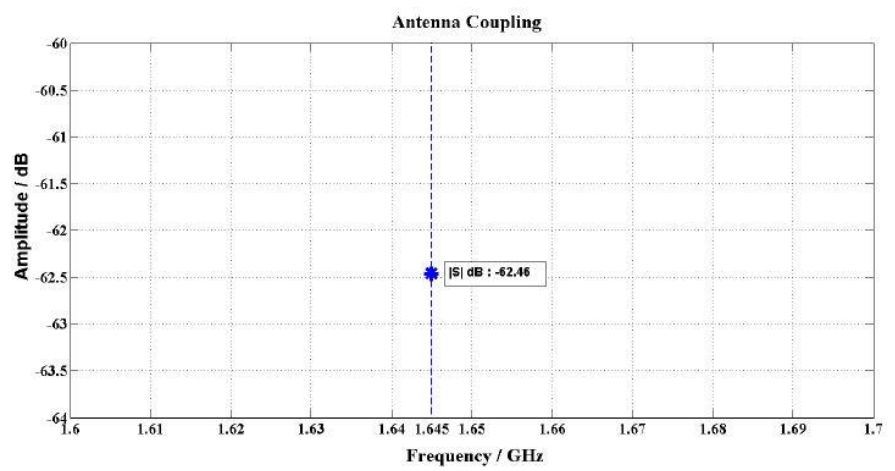

Fig. 6. Antenna coupling between INMARSAT C transmit antenna model and an element of the Iridium array satellite receive antenna model.

It should be noted that using this approach the antenna coupling is calculated at a single frequency, shown by a small star in Figure 6, at the operating frequency of the transmit antenna. The dashed blue vertical line was drawn to show the exact value of the coupling magnitude at $1645 \mathrm{MHz}$.

\section{VALIDATION OF SIMULATIONS USING SCALE MODELLING}

After obtaining simulated antenna coupling results, the key question would be whether those are representative of the actual measured antenna coupling results. To address this question validation of a number of simulations were performed using a 1:50 copper scale model of the Type 22 Batch II Frigate, as outlined in the following subsections.

Scale modelling of the EM characteristics of a system entails a scale construction of the conductive and EM features of the system [18] and it enables obtaining information about its EM behaviors [19].

For the purpose of this work a geometrical scale model, in which measurements were made on the model to just simulate the geometrical configurations of the lines of force in the fields of the full scale system, was considered to be sufficient. This can be contrasted to an absolute scale model which would also require the power of the full scale system to be simulated, since the model would then yield relative antenna coupling results comparable to those of the full scale system.

Geometric scaling is performed by substituting equivalent spatial coordinates to those of the full scale system for the physical scale model, as outlined below [18].

$$
\begin{gathered}
x=q x^{\prime} \quad y=q y^{\prime} \\
z=q z^{\prime} \quad t=\xi t^{\prime} \\
E(x, y, z, t)=\alpha E^{\prime}\left(x^{\prime}, y^{\prime}, z^{\prime}, t^{\prime}\right) \\
H(x, y, z, t)=\beta H^{\prime}\left(x^{\prime}, y^{\prime}, z^{\prime}, t^{\prime}\right)
\end{gathered}
$$

where

$x, y, z$ are the $3 \mathrm{D}$ spatial coordinate system 
$q$ is the physical or geometric scale factor

$t$ is the time

$\xi$ is the time scale factor

$E$ is the electric intensity

$H$ is the magnetic intensity

$\alpha$ is the scale factor for electric intensity

$\beta$ is the scale factor for magnetic intensity

In Equations (5) to (7) the quantities for the full scale system are given in the left hand sides while the quantities, including their appropriate scale factors, for the scale model are given in the right hand sides and are indicated by the symbol prime (').

In constructing a geometrical model, there are additional requirements that need to be satisfied. These requirements are outlined in Table I.

\section{TABLE 1}

THE RELATIONSHIP BETWEEN VARIOUS FULL SCALE AND MODEL QUANTITIES [18]

\begin{tabular}{|c|c|c|}
\hline Name of Quantity & Full Scale System & Model System \\
\hline Length & $l$ & $l^{\prime}=l / q$ \\
\hline Time & $t$ & $t^{\prime}=t / q$ \\
\hline Conductivity & $\sigma$ & $\sigma^{\prime}=q \sigma$ \\
\hline Permittivity & $\varepsilon$ & $\varepsilon^{\prime}=\varepsilon$ \\
\hline Permeability & $\mu$ & $\mu^{\prime}=\mu$ \\
\hline Frequency & $f$ & $\mathrm{f}^{\prime}=\mathrm{qf}$ \\
\hline Wavelength & $\lambda$ & $\lambda^{\prime}=\lambda / \mathrm{q}$ \\
\hline Phase Velocity & $v$ & $\mathrm{v}^{\prime}=\lambda / \mathrm{q}$ \\
\hline Propagation Constant & $\gamma$ & $\Upsilon^{\prime}=\mathrm{q}^{\prime} \Upsilon$ \\
\hline Resistance & $R$ & $\mathrm{R}^{\prime}=\mathrm{R}$ \\
\hline Reactance & $X$ & $\mathrm{X}^{\prime}=X$ \\
\hline Impedance & $Z$ & $\mathrm{Z}^{\prime}=Z$ \\
\hline Capacitance & $C_{p}$ & $C_{p}{ }^{\prime}=C_{p} / q$ \\
\hline Inductance & $I_{L}$ & $I_{L}{ }^{\prime}=I_{L} / q$ \\
\hline Antenna Gain & $G$ & $\mathrm{G}^{\prime}=\mathrm{G}$ \\
\hline
\end{tabular}

It should be noted that the permittivity and permeability are not an issue in this case because the choice of scaling factors makes them the same as in the case of full scale ship.

\section{A. Validation of Simulations using 1:50 Scale Models of the Type 22 Batch II Frigate}

A 1:50 physical copper scale model of the Type 22 Batch II Frigate was provided by QinetiQ at Funtington, UK with permission of UK Ministry of Defence. The model was 3 meters long and it is shown in Figure 7. It had a number of built-in whip antennas on it which were used to measure the antenna couplings between them.

Based on the dimensions of the 1:50 physical copper scale model, an equivalent 1:50 virtual scale model was produced using copper annealed in CST MWS.

Given the built-in whip antennas on the topside of the 1:50 physical scale model of the Type 22 Batch II Frigate, equivalent virtual antennas were produced and allocated to corresponding locations on the 1:50 virtual scale model, as shown in the bottom half of Figure 8.

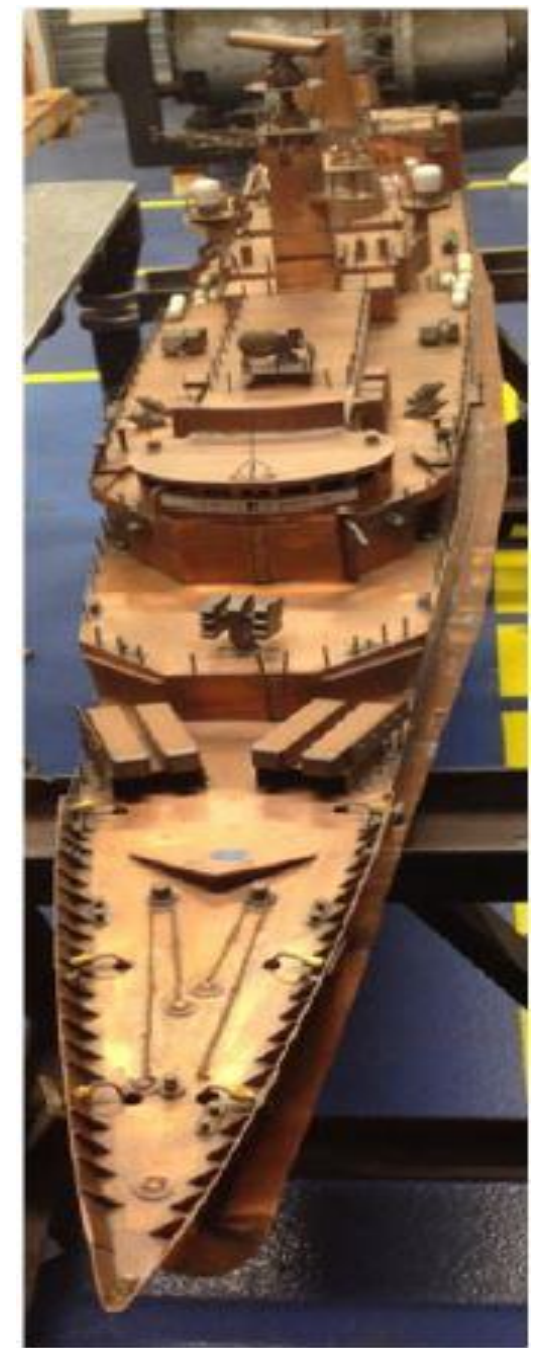

Fig. 7. 1:50 physical copper scale model for the Type 22 Batch II Frigate

For simplicity each of the built-in antennas was labelled as front, middle (mid-ships) and back (aft) whips, rather than using their designated UK MoD names.

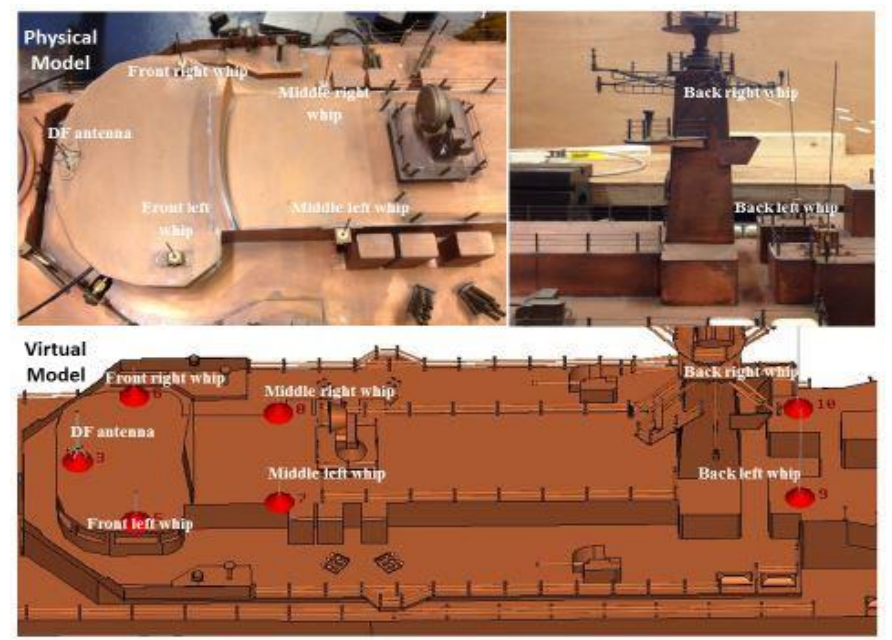

Fig. 8. Locations of the antenna pairs investigated on the 1:50 scale models of the Type 22 Bach II Frigate. 


\section{B. Antenna Couplings on the 1:50 Scale Models of the Type 22 Batch II Frigate}

The couplings between the antennas on the 1:50 physical scale model were measured using a Vector Network Analyzer which allowed the transmission and reception of EM energy between two antennas to be recorded in the form of Sparameters. The coupling for the same antennas on the 1:50 virtual model was computed in CST MWS.

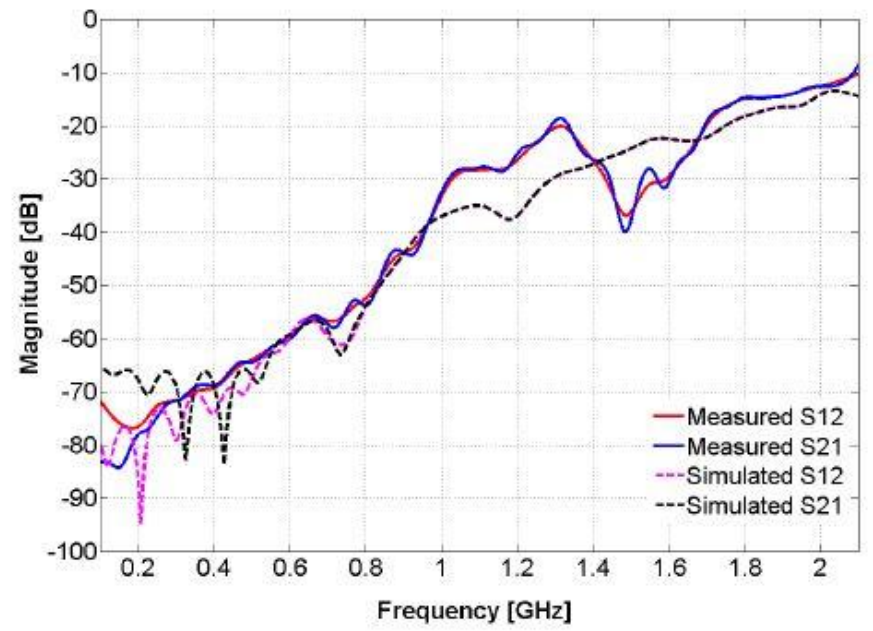

Fig. 9. Coupling comparison for mid-ships starboard vs midships port whip antennas, for 1:50 scale models of the T22 Batch II Frigate.

Once coupling results for both virtual and simulated models were obtained, they were co-plotted, for corresponding pair of antennas. The measured and simulated interaction between the two whip antennas located between the bridge and foremast is given in Figure 9. It can be seen that the two sets of results are very similar, especially at the operating frequencies of the two antennas $(1.885 \mathrm{GHz})$ where the variation is about $2 \mathrm{~dB}$.

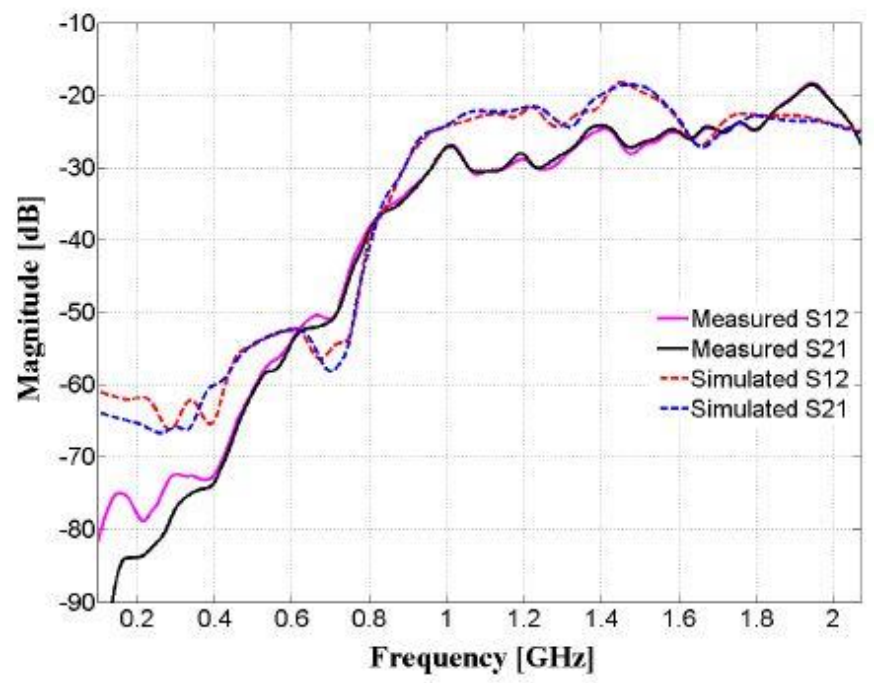

Fig. 10. Coupling comparison for mid-ships starboard whip antenna vs DF antenna.
The measured and simulated coupling result for starboard whip antenna, located between the bridge and foremast, and DF antenna is given in Figure 10. Again, the measured and simulated results resemble each other especially at the operating frequencies of the two antennas, namely $(0.88 \mathrm{GHz})$ and $(1.885 \mathrm{GHz})$ where the largest variation is about $5 \mathrm{~dB}$.

The coupling comparison plot between measured and simulated results for port whip antenna located on the bridge and port whip antenna located between the bridge and foremast is given in Figure 11. It can be seen that the measured and simulated coupling results closely resemble each other.

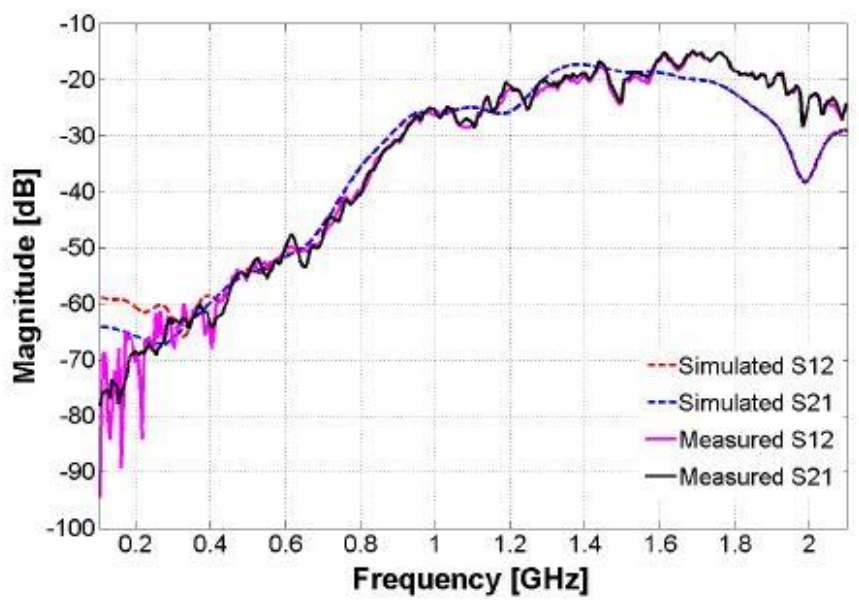

Fig. 11. Coupling comparison for front port vs mid-ships port whip antennas.

\section{CROSS VAliDATION}

In the previous section validation of a number of simulations were carried out using 1:50 physical and virtual scale models of the Type 22 Batch II Frigate. In all of those cases, the simulations were carried out in CST MWS using the T-Solver which allowed broadband coupling calculations. Based on the validation of the simulation results, it was deduced that simulations carried out in CST MWS using the T-Solver were a very good representation of these two scales given \pm 1 to 5 $\mathrm{dB}$ difference generally. However, the T-Solver is not suitable for simulating shipboard antenna models operating at upper UHF band or lower SHF band, such as the radar and satellite communication antenna models on the ship model. This is due to the enormous computational and memory requirements of the T-Solver at frequencies of over $500 \mathrm{MHz}$.

Since many of the shipboard EM sensors operate at frequency range of 1 to $12 \mathrm{GHz}$, the use of the T-Solver is not suitable for simulating the interference between such sensors. This is in part due to large Random Access Memory (RAM) requirements of a simulation, tens of gigabytes, and in part due to the time it takes to complete one simulation which may take one to several weeks - depending on the size and geometrical complexity of the structure, frequency range setting of the simulation and the speed of the computer processor. The computational and memory requirement were reduced by replacing the antenna models on the ship model with their equivalent far-field sources and then using either the 
I-Solver or A-Solver of CST MWS to calculate the antenna coupling between the excitation sources on the ship model. However, in order to acquire the required confidence on the latter method, it also needed to be validated. This was achieved by cross-validating the coupling results, comparing the coupling results obtained when utilising different antenna coupling computation methods to check whether similar results are obtained. The cross-validation was performed using coupling results obtained from I and A-Solvers of the CST MWS against the coupling results obtained when using the TSolver of the CST MWS, for the same antennas and their equivalent far-field sources. The cross-validations of the A and I-Solvers of CST MWS are further outlined below.

\section{A. Cross Validation of the I-Solver of the CST MWS against the T-Solver of the CST MWS}

The cross validation of the I-Solver of the CST MWS against the T-Solver of the CST MWS was performed by using two antennas: one a folded dipole antenna operating at $20 \mathrm{MHz}$ and another an end-loaded dipole antenna operating at 30 $\mathrm{MHz}$, on the Type 23 Frigate model with overall length of 133 meters.

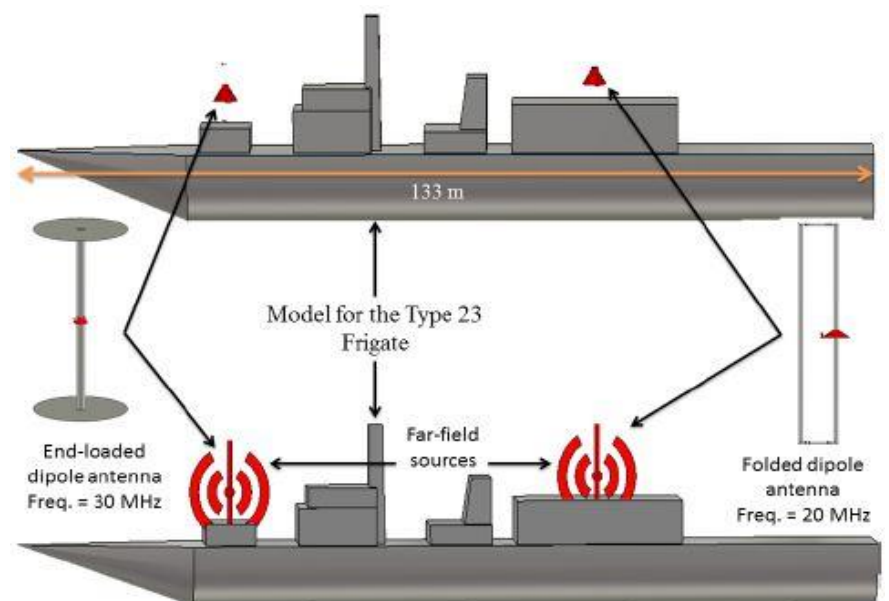

Fig. 12. Set up for cross-validation of the I-Solver of the CST MWS against the T-Solver of the CST MWS for a model of the Type 23 Frigate.

The two dipole antenna models were first produced in CST MWS, with the end-loaded dipole antenna model having its center frequency at $30 \mathrm{MHz}$ and the folded dipole antenna model having its center frequency at $20 \mathrm{MHz}$. The 3D far-field radiation pattern plot for each of the two antennas was then generated. The antenna models were imported on the Type 23 Frigate model and placed at two different locations on the ship model, as can be seen in Figure 12. A Time Domain simulation was then run on the combined frigate and antenna models and the coupling results are shown in Figure 13 by solid lines. The far-field excitation sources for the two antennas were imported on to an identical Type 23 Frigate model and placed in corresponding End-loaded dipole and folded dipole antenna positions on the ship model, as can be seen in the bottom part of Figure 12. The Integral equation solver of the CST MWS was utilized to run an I-Solver simulation on the combined ship and excitation sources for the antenna models. At the end of the simulation the resultant farfield sources for each of the two equivalent antenna sources were obtained. These, unlike the original far-field sources, took the effects of the structure into account. The resultant farfield sources were then used in the CST macro to calculate the antenna coupling. The results are shown by horizontal lines, both at 20 and $30 \mathrm{MHz}$, in Figure 13.

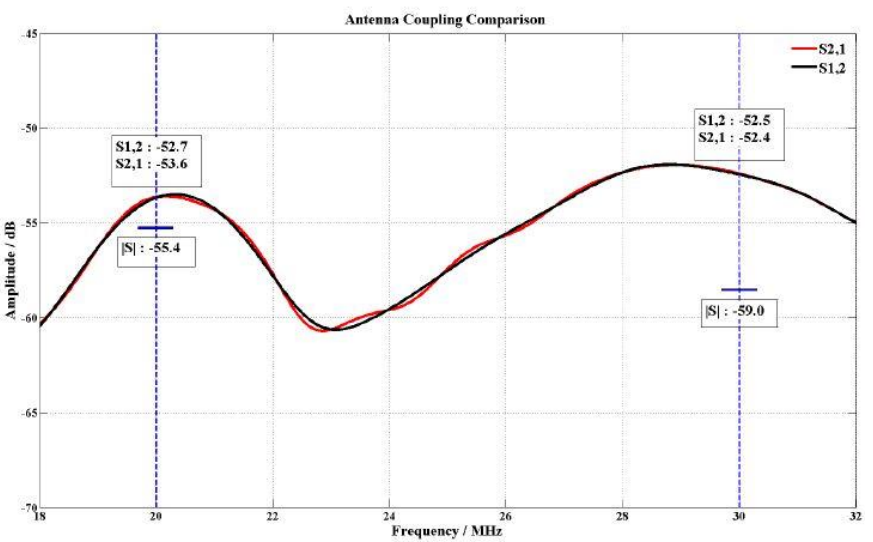

Fig. 13. Result for cross-validation of the I-Solver of the CST MWS against the T-Solver of the CST MWS.

It can be seen that the T-Solver of the CST MWS calculates the antenna coupling over broadband range of frequency while the I-Solver of the CST MWS calculates antenna coupling only at single frequencies. The level of coupling to the endloaded dipole antenna, which operates at center frequency of $30 \mathrm{MHz}$, when the folded dipole antenna is operating at center frequency of $20 \mathrm{MHz}$ transmits, based on Time Domain simulation, is $-52.5 \mathrm{~dB}$. Based on Integral Equation simulation, the same coupling is $-55.4 \mathrm{~dB}$. In this instance, the difference between the Time Domain based coupling calculation and the Integral Equation based coupling calculation is $2.9 \mathrm{~dB}$. The level of coupling to the folded dipole antenna when the end-loaded dipole antenna transmits, based on Time Domain simulation, is $-53.6 \mathrm{~dB}$. Based on Integral Equation simulation, the same coupling is $-59.0 \mathrm{~dB}$. In this latter instance, the difference between the Time Domain based coupling calculation and the Integral Equation based coupling calculation is $5.4 \mathrm{~dB}$. In both cases, difference between the two approaches can be considered to be plausible and probably sufficient for the early stages of ship design. Therefore, either of the two methods could be employed to calculate antenna couplings between shipboard antennas, located in the far-field of each other.

\section{B. Cross Validation of the A-Solver of the CST MWS against the T-Solver of the CST MWS}

Just like the I-Solver, the far-field excitation sources for the antennas can equally be used with the A-Solver of the CST MWS to calculate antenna coupling. The A-Solver can be several times faster than the I-Solver and therefore it is a good choice for simulating antenna models on the ship model. Like the I-Solver, the A-Solver of the CST MWS was cross validated against the T-Solver of the CST MWS to see 
whether the coupling results obtained, when using the ASolver, could be trusted.

The cross validation of the A-Solver against the T-Solver was performed by using two antenna models, a Ridged Pyramidal horn antenna having its center frequency at 1.8 $\mathrm{GHz}$ and a Biconical antenna having its center frequency at $1.5 \mathrm{GHz}$. This occurred with the antenna models being on a flat sheet of PEC with dimensions of $200 \mathrm{~cm} \times 50 \mathrm{~cm} \times 0.5$ $\mathrm{cm}$, as can be seen in Figure 14. The PEC sheet was chosen to be small so that it was possible to run Time Domain simulation on the combined structure and antenna models. The horizontal distance between the two antennas on the flat sheet of PEC was selected to be $144.7 \mathrm{~cm}$ and the two antennas were chosen to radiate EM energy towards the maximum reception direction of each other. Prior to placing the antennas on the structure, their 3D far-field radiation patterns were calculated and saved as far-field excitation sources. The saved far-field excitation sources were then imported on to an identical flat sheet of PEC and positioned in the locations of the corresponding antennas, shown in the bottom half of Figure 14.

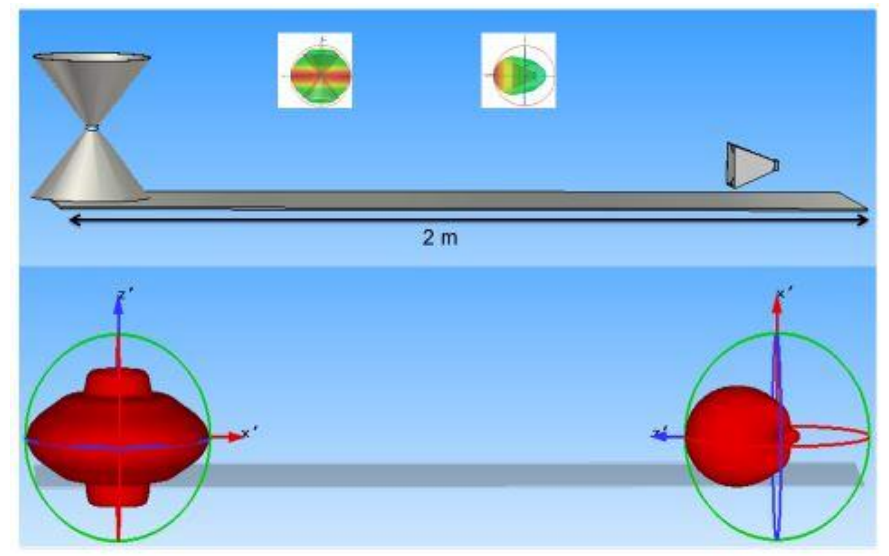

Fig. 14. Set up for cross-validation of the A-Solver of the CST MWS against the T-Solver of the CST MWS on a flat PEC sheet.

A Time Domain based simulation was run on the combined structure and antennas model and the antenna coupling result was recorded at the end of the simulation, which is given by the solid lines in Figure 15. An Asymptotic based simulation was run on the combined structure and excitation sources for antenna models. The coupling result for the latter case was recorded and it is given by horizontal lines in Figure 15.

The level of coupling to the horn antenna, operating at a center frequency of $1.8 \mathrm{GHz}$, when the Biconical antenna is operating at center frequency of $1.5 \mathrm{GHz}$, transmits, based on Time Domain simulation, is $-27.7 \mathrm{~dB}$. Based on Asymptotic simulation, the same coupling is $-27.2 \mathrm{~dB}$. In this instance, the difference between the Time Domain based coupling calculation and the asymptotic based coupling calculation is $0.5 \mathrm{~dB}$. The level of coupling to the Biconical antenna when the horn antenna transmits, based on Time Domain simulation, is $-27.1 \mathrm{~dB}$. Using the asymptotic simulation, the same coupling is given as $-28.8 \mathrm{~dB}$. In this latter instance, the difference between the Time Domain based coupling calculation and the asymptotic based coupling calculation is $1.7 \mathrm{~dB}$.

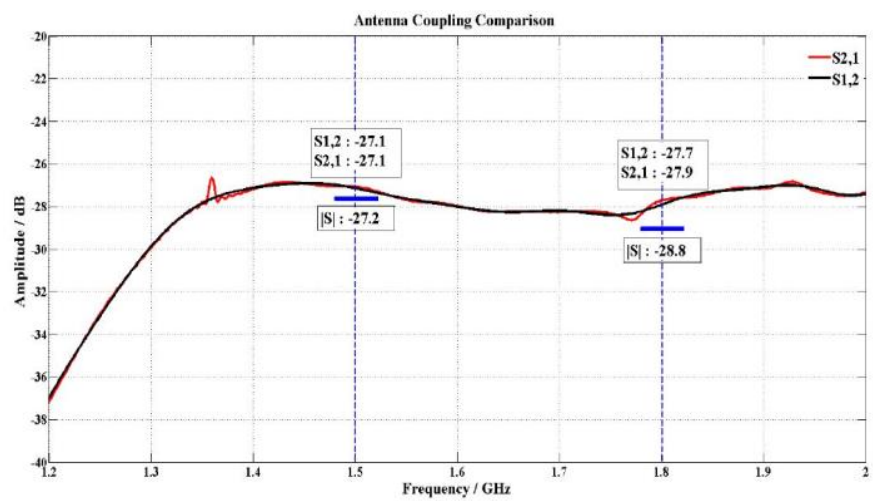

Fig. 15. Result for cross-validation of the A-Solver of the CST MWS against the T-Solver of the CST MWS for a horn antenna and a biconical antenna.

Having obtained very good agreement between the Time Domain based coupling calculation and asymptotic based coupling calculation using two antennas on a flat PEC sheet, it was important to compare the two methods by increasing the complexity of the structure. Thus, obstructions, such as a pentagon, a vertical flat sheet and a chamfered brick, between the antennas and their corresponding far-field sources were added to the flat PEC sheet, as can be seen in Figure 16.

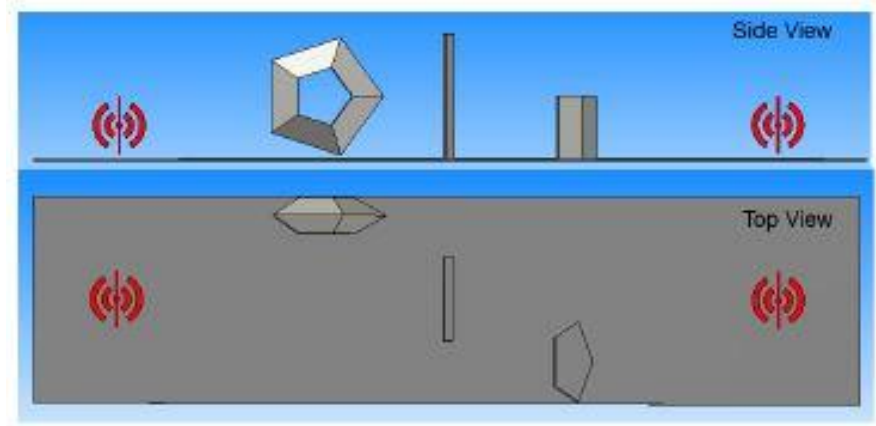

Fig. 16. Far-field excitation sources on a complex structure.

The addition of the obstructions blocked and reflected the EM waves emitted from each of the two different antennas and their equivalent far-field excitation sources in various directions. In addition, the obstructions have changed the reflection coefficients for the antennas, particularly affecting the horn antenna. Its $\mathrm{S} 11$ value, at $1.8 \mathrm{GHz}$, changed from $13.3 \mathrm{~dB}$ to $4.2 \mathrm{~dB}$ - making it highly inefficient at $1.8 \mathrm{GHz}$. Therefore, the operating frequency of the horn for crossvalidation purposes had to be changed from $1.8 \mathrm{GHz}$ to $2 \mathrm{GHz}$ at which frequency it had a $\mathrm{S} 11$ value of $-11.1 \mathrm{~dB}$. This implied that a new far-field excitation source for the horn antenna had to be modelled and then imported on to the complex PEC sheet.

The result of the Time Domain based coupling calculation is given by solid lines in Figure 17, while the result of the asymptotic based coupling calculations is given by horizontal lines in Figure 17. It should be noted that, given the 
computational resources available, the maximum frequency for simulating the new structure and antenna models was 2 $\mathrm{GHz}$.

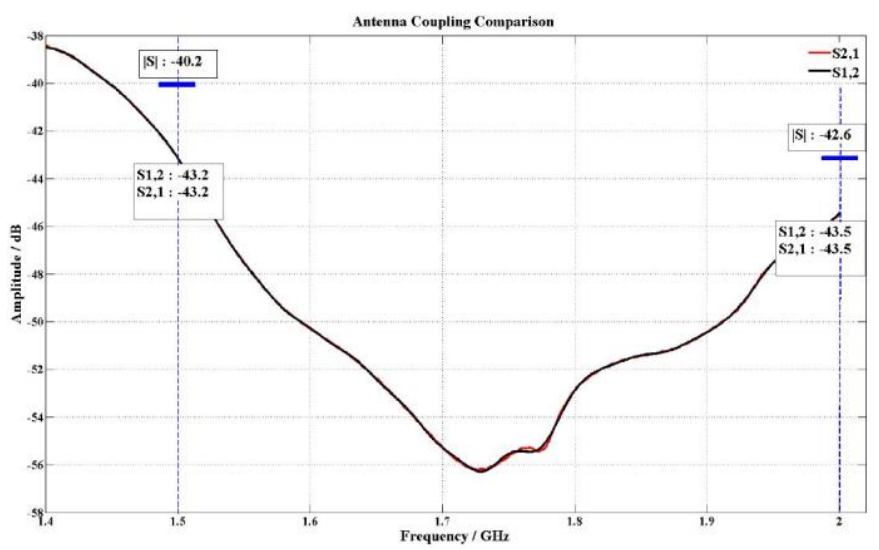

Fig. 17. Result for cross-validation of the A-Solver of the CST MWS against the T-Solver of the CST MWS when using complex structure.

The level of coupling to the horn antenna, operating at $2 \mathrm{GHz}$, when the Biconical antenna, operating at a center frequency of $1.5 \mathrm{GHz}$, transmits, based on Time Domain simulation, is $-43.5 \mathrm{~dB}$. Based on asymptotic simulation, the same coupling is $-40.2 \mathrm{~dB}$. In this instance, the difference between the Time Domain based coupling calculation and the asymptotic based coupling calculation is $3.3 \mathrm{~dB}$. The level of coupling to the Biconical antenna when the horn antenna transmits, based on Time Domain simulation, is $-43.2 \mathrm{~dB}$ while, based on asymptotic simulation, it is $-42.6 \mathrm{~dB}$. In this latter instance, the difference between the Time Domain based coupling calculation and the asymptotic based coupling calculation is $0.6 \mathrm{~dB}$. Therefore, the maximum difference between the two methods when using the complex structure is about $3.3 \mathrm{~dB}$ which is considered to be good for early stage ship design.

Having cross-validated simulations obtained from the I and A-Solvers of the CST MWS against the T-Solver of the CST MWS, it can be concluded that both I and A-Solver yield similar coupling results to that of the T-Solver, with the latter being the most accurate of the three. Therefore, whenever possible, the use of the T-Solver of the CST MWS is recommended over the others, for calculating antenna couplings.

\section{CONCLUSION}

This paper has shown how a commercially available CEM tool, such as CST MWS, can be utilized to predict the interactions of shipboard EM sensors in the concept phase of ship design. The interference between low frequency operating antennas can be determined by simulating the combined antennas and the ship's structure model using the TSolver of the CST MWS. The reliability of this approach was ascertained by obtaining similar results to those of the measured results, which were obtained using a 1:50 physical copper scale model for a Type 22 Batch II Frigate. The interference between shipboard antennas operating at UHF or above can be computed by simulating the equivalent far-field sources for the antennas on the ship model and then using either the I or A-Solver of the CST MWS to simulate the combined ship and antenna equivalent sources model. The fidelity of this approach was realized by cross-validating antenna coupling results, obtained when using both I and ASolvers of the CST MWS, against corresponding antenna coupling results, acquired using the T-Solver of the CST MWS, and getting comparable results with small differences.

\section{ACKNOWLEDGMENT}

The authors would like to thank the UK Ministry of Defence and University College London for supporting this research through an Impact Studentship.

\section{REFERENCES}

[1] P. E. Law, Shipboard Electromagnetics. Artech House, Dedham MA, 1987.

[2] A. Gharib, D. J. Andrews and H.D. Griffiths, "Topside Prediction of EMI Coupling for Early Stage Ship Design," Proc. Warship: Minor Warships, (Bath, UK), 2013.

[3] R. Adams, T. Harwood and M. Maiuzzo, "An innovative signal distribution system that allows EMI free communications for navy ships," Proc. MILCOM, (San Diego, CA), 2008, pp. 394 - 397.

[4] T. A. Adamson, Electronic Communications. 2nd ed., Kentucky: Delmar Cengage Learning, 1992.

[5] H. Griffiths, L. Cohen, S. Watts, E. Mokole and C. Baker, "Radar Spectrum Management and Engineering Issues -

Regulatory and Technical Approaches," IEEE Proc., vol. 103, no. 1, pp. 85-102, 2014.

[6] K. Malaric, EMI Protection for Communication Systems. Artech House, Dedham MA, 2010.

[7] M. A. Richards, J. A. Scheer and W. A. Holm, Principles of Modern Radar: Basic Principles vol. 1. SciTech Publishing Inc, Raleigh, 2009.

[8] H. D. Griffiths, C. J. Baker and D. Adamy, Introduction to Airborne Radar. 3rd ed., SciTech Publishing Inc, Raleigh NC, 2014.

[9] J. F. Garrett, R. L. Hardie and P. A. Rogers, "Let's design out EMI!," Naval Engineers Journal, vol. 94, no. 1, pp. 3740, Feb. 1982.

[10] D. Manley, "The loss of HMS Sheffield - a technical reassessment," Proc. Warship: Future Surface Vessels, (Bath, UK), 2015, pp. $129-139$.

[11] D. J. Andrews, "The true nature of ship concept design and what it means for the future development of CASD,"

Proc. COMPIT, (Cortona, Italy), 2013, pp. 33 - 50.

[12] N. T. Baron and D. R. Cebulski, "EMI - the enemy within," Naval Engineers Journal, vol. 104, no. 2, pp. 69-80, Mar. 1992.

[13] W. G. Duff, Designing Electronic Systems for EMC. SciTech Publishing Inc, Raleigh NC, 2011.

[14] H. W. Ott, Noise Reduction Techniques in Electronic Systems. $2^{\text {nd }}$ ed., Wiley \& Sons, New York, 1988.

[15] M. Blair and T. L. Stevens, Steel Castings Handbook. ASM International, 6th ed., 1995. 
[16] D. P. Johns. (2012, Sep.), "EMC/E3 Analysis of Rotorcraft Electrical System.” [Video Online]. Available: https://www.youtube.com/watch?v=i3rX_5lmpEU

[17] E. Tanjong. (2010, Nov.), "Modelling the installed performance of antennas in a ship topside environment." [Online]. Available: https://www.cst. com/Content/Events/Event item_1731/CST_Installed performance.pdf.

[18] A. L. Whitson, "Electromagnetic dimensional scale modeling," Stanford Research Institute, Stanford, 1974, pp. $201-211$.

[19] G. Sinclair, "Theory of models of electromagnetic systems," Proc. IRE, 1948, pp. 1364 - 1370.

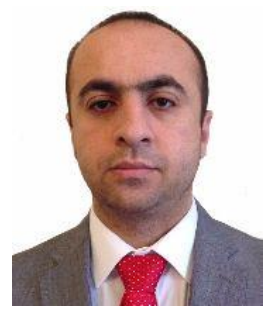

Ajmal Gharib gained his MEng degree in Systems and Control Engineering from the University of Sheffield UK in 2010 and his $\mathrm{PhD}$ degree from University College London UK in 2015. After finishing his $\mathrm{PhD}$ degree, he has worked as a Data Scientist, on a knowledge transfer programme, in the Department for Business, Innovation and Skills, evaluating the impact of science and research in the UK. His research interests include radar, antenna and electromagnetic

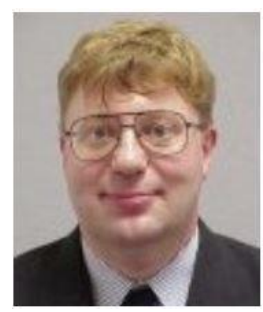

Hugh D. Griffiths (M'86, SM'90, F'99) holds the THALES/Royal Academy of Engineering Chair of RF Sensors at UCL. $\mathrm{He}$ received the IERE Lord Brabazon Premium Award in 1984, the IEE Mountbatten and Maxwell Premium Awards in 1996, the IEEE Nathanson Award in 1996, and the IET A F Harvey Research Prize in 2012. He served as President of the IEEE AES Society for 2012 - 2013, and serves as Editor-in-Chief of the journal IET Radar, Sonar and Navigation. He is a Fellow of the IET, Fellow of the Institute of Acoustics and of the IEEE, and in 1997 he was elected to Fellowship of the Royal Academy of Engineering.

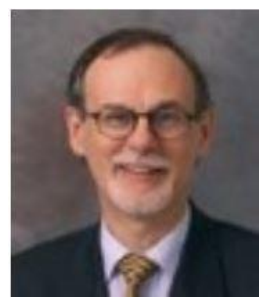

David J. Andrews was appointed Professor of Engineering Design at UCL in September 2000 following an extensive career in ship design and acquisition management in the UK Defence Procurement Agency, including managing a range of ship concept designs. He leads UCL's design research in computer aided ship design, design methodology and design practice. He is a Fellow of RINA, Fellow of IMechE and was elected to the Royal Academy of Engineering in 2000 and served as a Vice President of the Royal Institute of Naval Architects in 2006. engineering, and analytics of sensor data. 